\title{
AN ENCLOSURE THEOREM FOR EIGENVALUES
}

BY H. D. BLOCK AND W. H. J. FUCHS

Communicated by R. P. Boas, May 8, 1961

THEOREM 1. Let $H$ be a hermitian matrix and $x$ an arbitrary vector of unit length. Let $\mu=(H x, x), \sigma=\left(\|H x\|^{2}-\mu^{2}\right)^{1 / 2}$. Then there is an eigenvalue of $H$ in the interval:

$$
\mu-\sigma \leqq \lambda \leqq \mu+\sigma .
$$

REMARK. The quantity under the radical is non-negative, since

$$
\mu^{2}=|(H x, x)|^{2} \leqq\|H x\|^{2} .
$$

Theorem 1 is a special case of the following theorem.

THEOREM 2. Let $H$ be a matrix having a complete orthonormal set of eigenvectors. Let $x$ be a vector of unit length. Let $\mu=(H x, x)$, $\sigma=\left(\|H x\|^{2}-|\mu|^{2}\right)^{1 / 2}$. Then there is an eigenvalue of $H$ in the circle: $|\lambda-\mu| \leqq \sigma$.

PRoof. Let $x=\sum \xi_{i} e_{i}$, where $H e_{i}=\lambda_{i} e_{i}$ and $\left(e_{i}, e_{j}\right)=\delta_{i j}$. Thus $(x, x)$ $=\sum\left|\xi_{i}\right|^{2}=1, \quad$ and $\sigma^{2}=((H-\mu I) x, \quad(H-\mu I) x)=\sum\left|\lambda_{i}-\mu\right|^{2}\left|\xi_{i}\right|^{2}$ $\geqq\left|\lambda_{m}-\mu\right|^{2}$, where $\left|\lambda_{m}-\mu\right|=\min _{i}\left|\lambda_{i}-\mu\right|$. Q.E.D.

Theorem 1 furnishes a simple device for obtaining an interval containing an eigenvalue. As $x$ approaches an eigenvector the interval length $(2 \sigma)$ approaches zero.

This method may be compared with Vazsonyi's enclosure method ${ }^{1}$ in which, for a symmetric matrix $H$, an eigenvalue is guaranteed to lie in the interval

$$
\min _{i} \mu_{i} \leqq \lambda \leqq \max _{i} \mu_{i}
$$

where $\mu_{i}$ is the ratio of the $i$ th component of $H x$ to the $i$ th component of $x$. It is an easy exercise to show that $2 \sigma \leqq\left[\max _{i} \mu_{i}-\min _{i} \mu_{i}\right]$. In general our interval is considerably smaller than the one obtained by the Vazsonyi method.

The method of Kohn and Kato guarantees, for a symmetric matrix, that an eigenvalue $\lambda_{p}$ lies in the interval

$$
\mu-\frac{\sigma^{2}}{\lambda_{p+1}-\mu} \leqq \lambda \leqq \mu+\frac{\sigma^{2}}{\mu-\lambda_{p-1}},
$$

${ }^{1}$ S. H. Crandall, Engineering analysis, New York, McGraw-Hill, 1956. 
where $\lambda_{p-1}<\lambda_{p}<\lambda_{p+1}$ are successive eigenvalues and $\lambda_{p-1}<\mu<\lambda_{p+1}$. For application of our method this information need not be available.

For purposes of numerical computation, fewer operations are required if one does not normalize $x$ in Theorem 1 at the outset, but instead defines $\mu=(H x, x) /\|x\|^{2}, \sigma=\left[\left(\|H x\|^{2} /\|x\|^{2}\right)-\mu^{2}\right]^{1 / 2}$.

CORNELL UnIVERSITY

ENGINEER
or
MATHEMATICIAN
The Radiation Laboratory
of the Johns Hopkins University
Has a Position for an
Engineer or
Applied Mathematician
experience in the field of:
SIGNAL DETECTION
AND ANALYSIS
With Emphasis on Sequential
Detection and Characterization
of Complex Signals
Favorable Arrangements for
Advanced Study in the
University Graduate Schools.
Excellent Laboratory Facilities
Broad Opportunity for
Career Development
Address Inquiries To:
RADIATION LABORATORY
THE JOHNS HOPKINS
UNIVERSITY
Homewood Campus
Baltimore I8, Md.

\begin{tabular}{|} 
MEMOIR 38 \\
TORSION FREE GROUPS \\
OF RANK TWO \\
by \\
R. A. BEAUMONT and \\
R. S. PIERCE \\
This Memoir is a report on investi- \\
gations of torsion free abelian groups \\
of rank two. The principal motivation \\
for this work is the authors' convic- \\
tion that detailed study of a nontrivial \\
class of torsion free groups is needed \\
as a basis for conjectures on arbitrary \\
torsion free groups. The first part of \\
the paper is devoted to establishing a \\
system of invariants for rank two \\
groups; the purpose of the last part of \\
the paper is to demonstrate the useful- \\
ness of these invariants. \\
4l pages \\
25\% discount to members \\
AMERICAN MATHEMATICAL \\
SOCIETY \\
190 Hope Street \\
Providence 6, Rhode Island \\
\hline
\end{tabular}

